\title{
Candidiasis Diagnosed at the University Hospital of Constantine, Algeria
}

\section{Djaballah M*, Fendri AH, Beldjoudi W, Rikhouah H, Ahrow S, Allouache B and Belmezdad A}

Laboratory of Parasitology and Mycology Constantine Hospital University, Constantine, Algeria

${ }^{*}$ Corresponding author: Djaballah M, Laboratory of Parasitology and Mycology Constantine Hospital University, Constantine, Algeria, E-mail: malikad887@gmail.com

Citation: Djaballah M, Fendri AH, Beldjoudi W, Rikhouah H, Ahrow S, et al. (2015) Candidiasis Diagnosed at the University Hospital of Constantine, Algeria. J Immunol Infect Dis 2(2): 201. doi: 10.15744/23946512.2.201

Received Date: February 18, 2015 Accepted Date: April 28, 2015 Published Date: April 30, 2015

\begin{abstract}
Introduction: Candida species are ubiquitous fungi and the most common fungal pathogens affecting humans. The aim of this study were to evaluate the epidemiology and describe the characteristics of patients with documented infections superficial or invasive caused by Candida species isolates in a Constantine teaching hospital and determine the risk factors.

Methods: From January 2012 to December 2012 all consecutives patients who developed candida At Constantine university hospital, were enrolled. Underlying diseases, clinical severity, age, gender, species distribution and risk factors including systematic disease, were analyzed. The diagnosis of candidiasis is confirmed by observation of mycelial forms on microscopic examination. Samples recruited are analyzed by direct examinations cultures and identification with Auxacolor-test.

Results: A total of 126 candida were identified. The proportion of candida albicans species $64 \%, 7 \%$ other species including C. tropicalis, C. glabrata, C. parapsilosis and C. dubliniensis) and C non albicans $28.5 \%$ could not be identified. The onyxis candidiasis represents the majority of diseases diagnosed with a rate of $37.3 \%$ followed by digestion with $35.70 \%$. The sex ratio was 0.36 . Of the 10 blood cultures received only 3 were positive.

Conclusion: The epidemiology of candidiasis in Constantine is associated with comorbidity and mortality with patients hospitalized in intensive care units and with high risk factors lesion witch necessity identification and taking in charge rapid Prophylaxis is a general point to prevent an infection.

Notably in candidiasis, it is very effective and based on risk factor identification. However, prophylaxis must be restricted to carefully selected groups of patients, to avoid the emergency of resistant strains and a shift in the distribution of pathogens from albicans to nonalbicans strains under the pressure of antifungal agents.

Keywords: Candida; Candidiasis; Mycose; Algeria
\end{abstract}

\section{Introduction}

Candidiasis are cosmopolitan mycoses caused by Candida yeasts. Different types of infections are correlated with Candida. Superficial (skin appendages and mucous membranes) and deep (septicemia and organ damage). Candidiasis is overwhelmingly opportunistic fungi that grow in the favor of local or general predisposing factors. The pathogens are represented by a multilateral budding yeast, unencapsulated, some produce filaments the others do not. In Culture they appear in the form of white colonies that are creamy non-pigmented.

Candida albicans (C. albicans) is the main species involved in human disease; other species are less frequently isolated (C. glabrata, C. parapsilosis, C. krusei). The genus of Candida albicans (usually with saprophytes, in the gastrointestinal tube and vaginal mucous), and sometimes by other types of Candida (C. parapsilosis, C. tropicalis, C. stellatoides etc.), affecting, in isolation or jointly, the mucosas, skin, nails and on occasion, other organs. It is of universal distribution, common in newborns, though it can also affect adults and elderly people. Some professionals, such as maids, dish washers, cooks and nurses, are more prone. Esophageal candidiasis is defining pathologies of AIDS. The clinical conditions are:

Oral candidiasis: It is more common in newborns and the elderly, debilitated patients and AIDS sufferers. Lesions are erosive and whitish in color.

Intertriginous candidiasis: It affects the natural folds (interdigital, inframammary, ungual, axillary) and is characterized by erosive, fissural, humid lesions, with a whitish, itchy coating, and by small, round, erythematous-squamous, satellite lesions, and even a bacterial postules.

Ungual and periungual candidiasis: These infections entail intense erythematous-squamous, periungual lesions (paronychia), which are painful and can lead to onycholysis. Typically, they affect the proximal edge of the nail and appear mainly on fingernails, since they have the most contact with water. 
Perleche (angular chelitis): This involves fissures at the corners of the mouth, which are common following changes in the architecture of the mouth following prosthesis and thus, it is common in elderly people. There is also a high incidence in newborns (there have been suspicions of vaginal contamination) [1].

Candidiasis usually is a result of damage in the epithelial barrier function. It occurs almost exclusively in debilitated patients whose normal defense mechanisms are impaired. A patient with cancer may be immunocompromised because of the underlying malignancy or anticancer therapy. Specific malignancies may be associated with immune deficit that predisposes to infection with a particular pathogen. Therapy with corticosteroids or cytotoxic drugs and localized or widespread irradiation result infurther deterioration of the host defense mechanism [2].

The first step of the treatment is the elimination of predisposing factors and Therapy for cutaneous candidiasis is dominated by topical antifungal agents. Azole antifungal cream (e.g., Bifonazole, Ketoconazole, Lanoconazole) is most effective. Terbinafine hydrochloride and amorolfine hydrochloride are also useful. Cutaneous candidiasis usually requires a shorter duration of topical treatment. For candidal paronychia and onychomycosis, oral therapy with itraconazole is recommended. The daily dose of itraconazole should be taken for several months. Itraconazole oral solution is commonly used for oral candidiasis, and miconazole gel is also effective.

Systemic treatment should be applied for extensive forms, for which fluconazole, itraconazole ketoconazole and amphotericin B, can be used [3].

Amphotericin B-based preparations, the azole antifungal agents, and the echinocandin antifungal agents play a role in treatment. Choice of therapy is guided by weighing the greater activity of amphotericin B-based preparations and the echinocandin antifungal agents for some non-albicans species (e.g., Candida krusei) against the ready availability of oral and parenteral formulations for the azole antifungal agents. Flucytosine has activity against many isolates of Candida but is infrequently used [4].

\section{Methods}

It is a retrospective and descriptive study. In 2012 we collected samples from different health facilities and prophylaxis of outpatient clinics. In superficial candidiasis, the specimens obtained for laboratory identification were urine, oral cavity, blood, sputum, broncho-alveolar liquid (BAL), stool, skin and nails wet mount smears use scrapings or smears examined under the microscope to identify hyphae, pseudohyphae, or budding yeast cells. Potassium hydroxide, lactophenol, or methylene blue stain may help directly demonstrate fungal cells. For each sample we made: direct examination, culture on Sabouraud agar and incubated at 37 ${ }^{\circ} \mathrm{C}$ for $48 \mathrm{~h}$. The plates were incubated at $30^{\circ} \mathrm{C}$ for $48 \mathrm{~h}$. Each morphological colony isolate was characterized according to [5]. After this period Germ-tube test Yeast cells were inoculated into $0.5 \mathrm{~mL}$ of fetal bovine serum and incubated at $37^{\circ} \mathrm{C}$ for $3 \mathrm{~h}$ than, aliquots were removed for microscopic examination [6]. Germ tube was considered as a slender tube with straight walls, without septum and without constriction at the junction between the cells [7]. Germ -tube was indicative of C. albicans or C. dubliniensis. Chlamydoconidia production test was performed using Agar potato-carrot medium ( $20 \mathrm{~g}$ potato $20 \mathrm{~g}$ carrot, $20 \mathrm{~g}$ bacteriological agar, and distilled water to a final adjusted volume of $1000 \mathrm{~mL}$ ). The samples previously grown in SDA were seeded as 3 parallel streaks in a rectangular piece of Potato agar placed between two slides, incubated in wet chamber at $30{ }^{\circ} \mathrm{C}$ for $72 \mathrm{~h}$ and visualized in an optical microscope (10X and 40X magnification). The formation of rounded spores with double-wall isolates was observed as chlamydoconidia, and was indicative of C. albicans or C. dubliniensis [8]. For other species we use The AUXACOLOR TM 2 kit to identify them which is system based on the principle of sugar assimilation [9]. The growth of yeasts is visualized by the colour change of a $\mathrm{pH}$ indicator. The kit also includes 3 enzyme tests, including a test for detection of the phenoloxidase activity of Cryptococcus neoformans.

The kit comprises:

- A negative control to facilitate interpretation of the sugar assimilation results (blue well).

- 13 sugar assimilation results, corresponding to the following sugars:

- Glucose (GLU.) : Positive control

- Maltose (MAL.) - Cellobiose (CEL.)

- Sucrose (SAC.) - Trehalose (TRE.)

- Galactose (GAL.) - Adonitol (ADO.)

- Lactose (LAC.) - Melezitose (MEL.)

- Raffinose (RAF.) - Xylose (XYL.)

- Inositol (INO.) - Arabinose (ARA.)

Each sugar is dehydrated in the presence of a basic solution and a $\mathrm{pH}$ indicator, bromocresol purple. The growth of a yeast is indicated by the colour change of the indicator from blue to yellow and by a cloudy appearance in the well. An enzymatic test to detect $\mathrm{N}$-acetyl-galactosaminidase activity (hexosaminidase: HEX). A positive reaction corresponds to a yellow colour of the well, while a negative test remains colourless.

- A phenoloxidase (POX.) test for detection of the phenoloxidase activity of Cryptococcus neoformans associated with a test to detect prolinearylamidase activity (PRO.): 
- A brown colour of the well indicates positive phenoloxidase (POX) activity.

- A yellow colour indicates positive prolinearylamidase (PRO) activity.

- Absence of coloration or grey coloration indicates a negative reaction for both tests.

The coexistence of the POX and PRO tests in the same well can be justified by the fact that these two tests are never both positive at the same time. The only possible profiles are POX negative/PRO negative; POX positive/PRO negative; POX negative/PRO positive with the colorimetric interpretation described above. AUXACOLORTM 25651320 tests COLORIMETRIC SUGAR ASSIMILATION TEST FOR IDENTIFICATION OF THE MAIN YEASTS OF MEDICAL INTEREST IVD BIORAD. Sanofi, Diagnostics Pasteur, France.

\section{Results}

In total, 126 strains of Candida were isolated in patients in the year 2012 study. The most frequently isolated Candida species were Candida albicans (C. albicans, (81strains, 64\%), followed by C. glabrata (2 strains, 1.58\%), C. tropicalis (3 strains, 2.38\%), C. parapsilosis (2 strains, 1.58\%), and C. dubliniensis (1 strain, 0.79\%) (Table 1) When analyzed on a patient basis. We found candidiasis predominant in female with $73 \%$. The modal class is between $30-40$ years (Table 1).

\begin{tabular}{|c|c|c|}
\hline Age groups (year) & No. of subjects & $\%$ \\
\hline $0-10$ & 18 & 14,2 \\
\hline $11-20$ & 7 & 5,5 \\
\hline $21-30$ & 17 & 13,4 \\
\hline $31-40$ & 32 & 25,3 \\
\hline $41-50$ & 21 & 16,6 \\
\hline $51-60$ & 17 & 13,4 \\
\hline$>60$ & 14 & 11,1 \\
\hline Gender & & \\
\hline Male & 34 & 23 \\
\hline Female & 92 & 73 \\
\hline Species isolated & & \\
\hline C. albicans & 81 & 64,2 \\
\hline C. glabrata & 2 & 1,5 \\
\hline C. tropicalis & 3 & 2,3 \\
\hline C. parapsilosis & 2 & 1,5 \\
\hline C. dubliniensis & 1 & 0,7 \\
\hline C. non albicans & 36 & 28,5 \\
\hline
\end{tabular}

Modal class is between 30 and 40 year, candidiasis was found predominant in female with $73 \%$. The modal class is between $30-40$ years Table 1: Distribution of Candida species isolated from patients with candidiasis in the year 2012 study According to age and gender

The auxacolor $28.5 \%$ could not be identified because of lack of reagents. According to the nature of sampling Woman's fingernails are the most common localization with a rate of $37 \%$ (Figure 1), followed by the digestive reached $34 \%$, vaginal mucosa 10 , urines 8, skin 7, bonchoalveolar and BAL 5 and blood culture 3. Some of the patients had immunodepressive pathologies (Figure 2). Among them we note in order of frequency: diabetes, HIV, patients service of anesthesia-reanimation no neglecting hematologic malignancies (Non Hodgkin Lymphoma, bone Marrow transplant) the number of positive. Patients and those with risk factors were 16 out of 22 with a rate of $72 \%$ (Figure 3 ).

\section{Discussion}

Candidiasis is an infection caused by a yeast-like fungus called Candida. It can infect the mouth, vagina, skin, stomach, and urinary tract. About $75 \%$ of women will get a vaginal yeast infection during their lifetime, and $90 \%$ of all people with HIV/AIDS develop candida infections. Oral infections, called oral thrush, are most common in infants, elderly people, and those with a weakened immune system [9]. Candidiasis are common conditions, but most often benign in absence of immunosuppression. Candidiasis is increasing in occurrence and severity because of advances in modern medicine and due to the indiscriminate use of antibiotics, immunosuppressive and cytotoxic therapies, and immunosuppressive situation, particularly in cases of granulocytopenia below 1000 elements / $\mathrm{ml}$ and more recently in AIDS. In this study we identified 126 cases with a rate of about $12 \%$, with a female predominance $73 \%$. The modal class is between $30-40$ years. The auxacolor allowed isolation $64 \%$ of C. albicans, $7 \%$ other species (C. tropicalis, C.glabrata, C.parapsilosis and C. dubliniensis) and 28.5\% non albicans could not be identified because of lack of reagents. Woman's fingernails are the most common localization with a rate of $37 \%$ (because of housework), followed by the digestive site reached $34 \%$. 


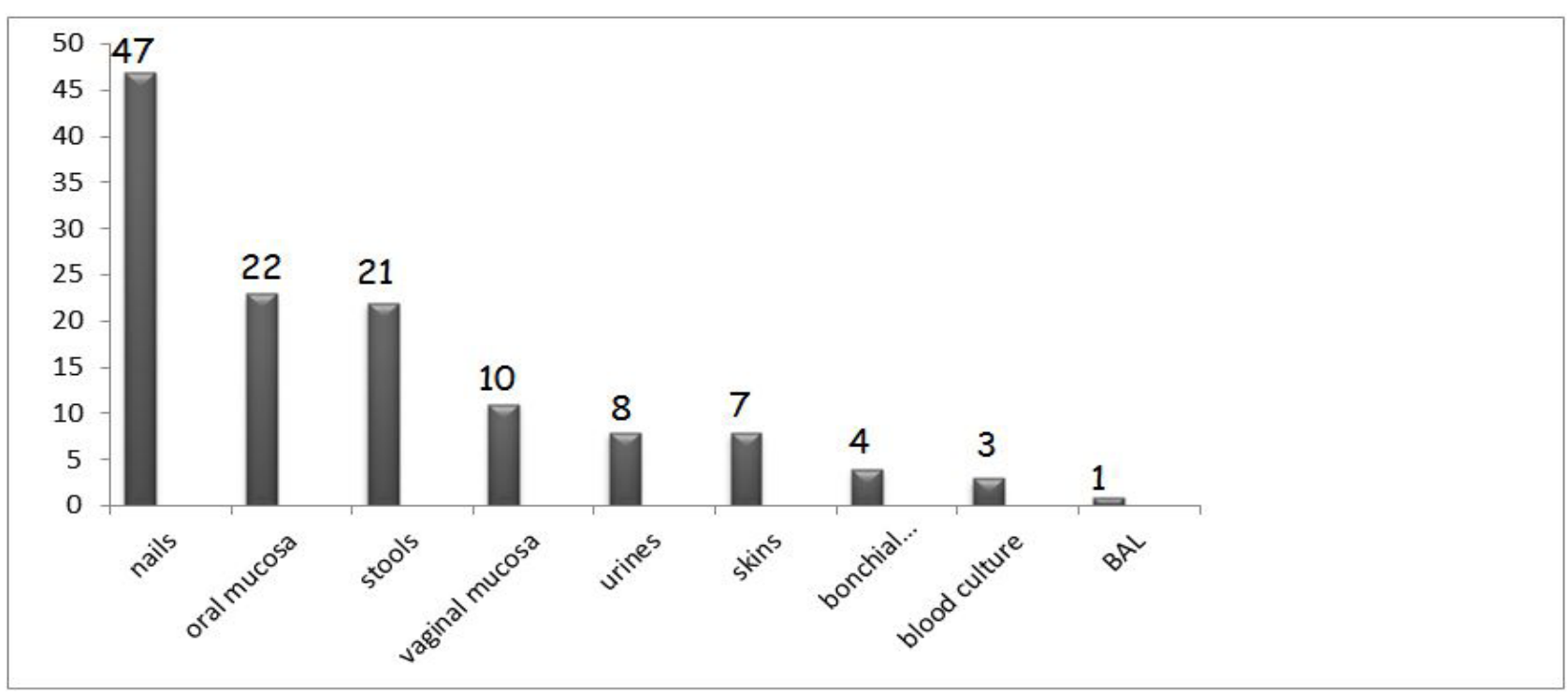

Woman's fingernails are the most common localization with a rate of $37 \%$ (because of housework) Figure 1: Cases According to the Nature of Sampling

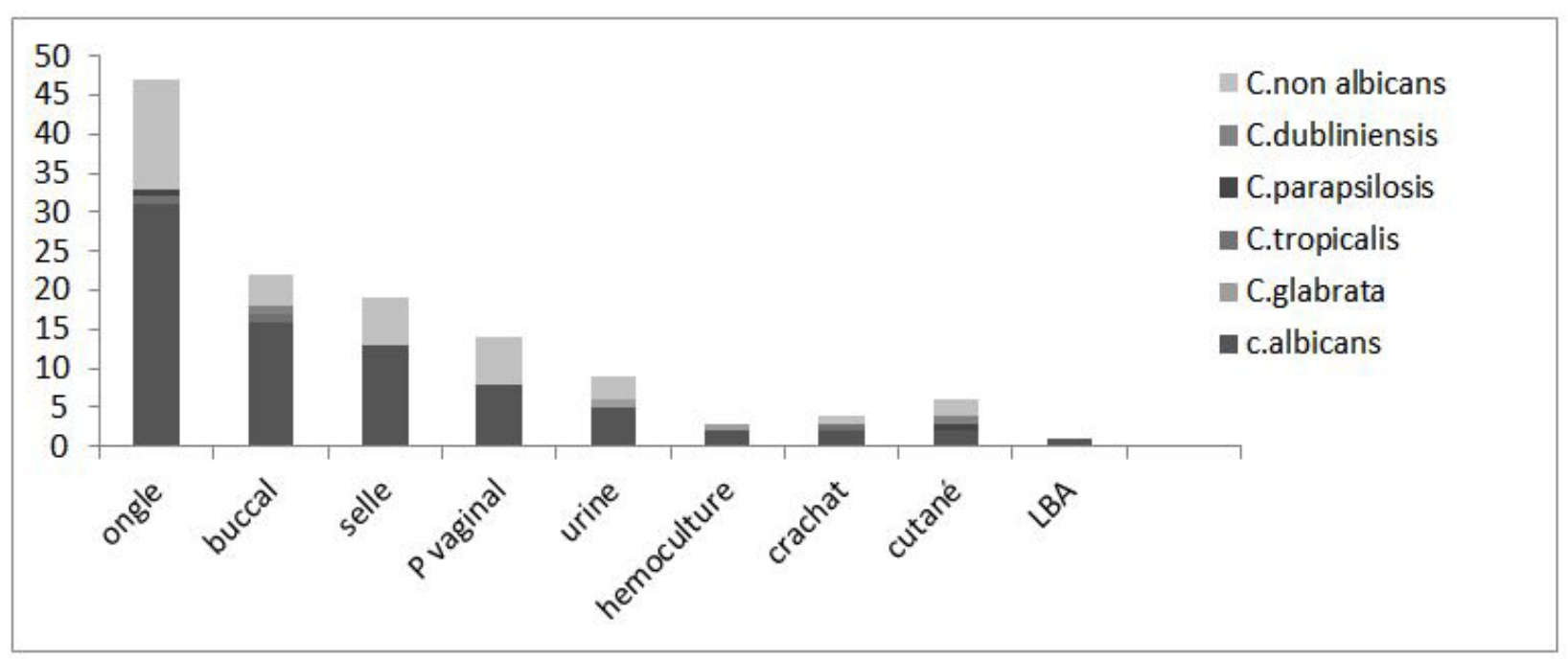

Candida albicans is the most specie isolated in all samples

Figure 2: Species Isolated in Terms of Localization

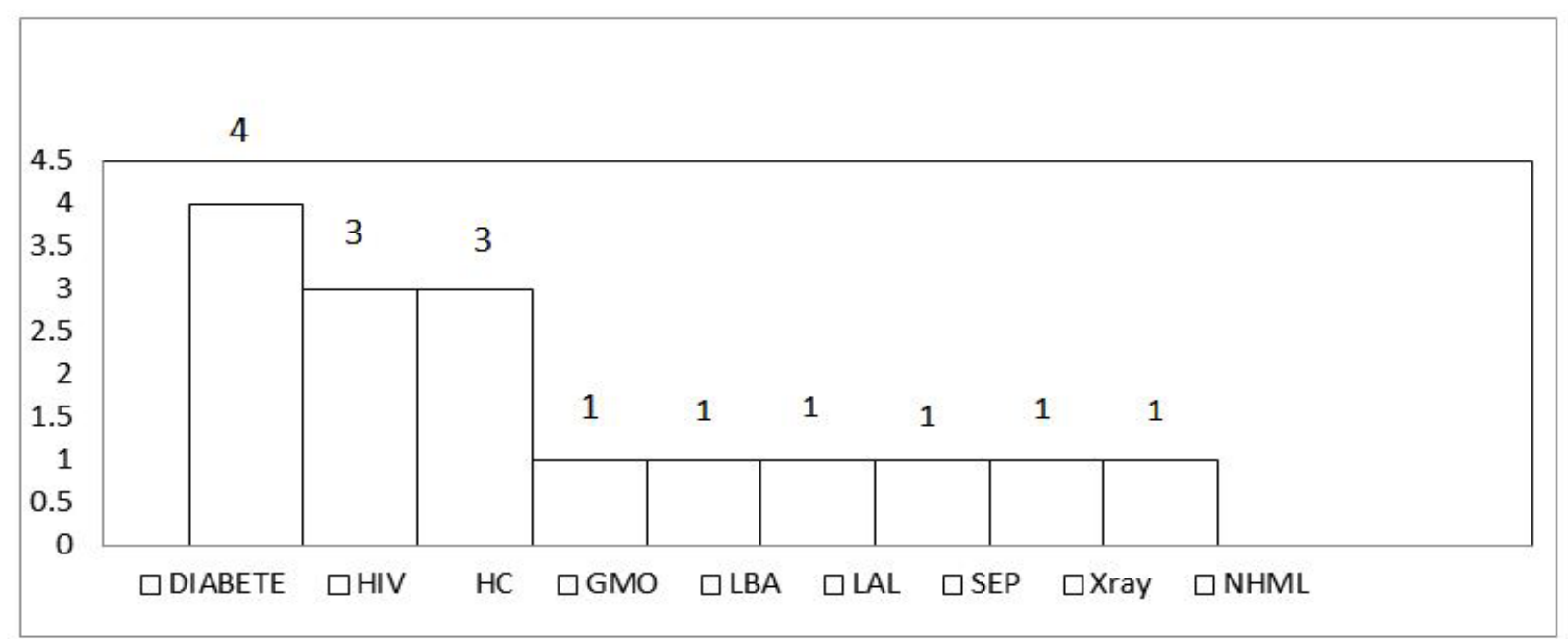

Figure 3: Candidiasis cases based on risk factors. Diabete; HIV: Human imunodeficience virus; Blood stream; GMO: Bone marow transplant; LAL: Lymphoblastic leukemia acute; SEP: Multiple sclerosis; NHML: Non Hodgkin lymphoma 
For urinary tract 8 (6.34\%) Candida species had been isolated Candiduria is commonly encountered in hospitalized patients, particularly those with a urinary catheter. Candiduria is indicative for a wide spectrum of conditions which may or may not require treatment. Asymptomatic candiduria has been followed long term, but no adverse consequences have been described [10]. Some of the patients had immunodepressive pathologies. Among them we note in order of frequency: diabetes, HIV, patients service of anesthesia-reanimation not neglecting hematologic malignancie (Non Hodgkin lymphoma, bone marrow transplant). The number of positive patients and those with risk factors is 16 out of 22 with a rate of $72 \%$. Of the 10 blood cultures received only 3 were positive. However in a national surveillance conducted in the USA between 1986 and 1990, Candida species were the fifth most common isolates to be recovered from blood cultures taken from hospital in-patients, and the fourth most commonly recovered pathogen (from all sites) in intensive care units. Candida species are becoming nearly as common a cause of hospital acquired infection as the more familiar bacterial pathogens. By far the commonest species to cause clinical infection is C. albicans [11]. In this study we found that candidiasis is increasing, in fact by comparing our results to a study by the same laboratory in 2004 (not published), which has noted the rate of 6\%. This rise is due to immunosuppressive therapies and the emergence of resistant candida species where the need is early diagnosis and treatment for better management. The most important prevention method for Candidiasis, and many other types of infections, is personal hygiene. A person should practice oral hygiene daily and bathe on a regular basis drying well when done. Use gentle soaps on the body, and avoid using fragrant soaps or body washes, which can cause irritations, especially in the genital areas. Scented tampons and feminine sprays should also be avoided. Another key to prevention is trying to reduce exposure of the hands to chemical soaps and water by wearing gloves while cleaning. Most of all, educating oneself on the infection at hand is the best way to prevent reoccurrence.

For diagnosis of invasive candidiasis, laboratory studies are nonspecific and lack sensitivity. In candidemia and disseminated candidiasis, blood cultures are helpful, but they are positive in only $40 \%$ to $60 \%$ of cases of disseminated disease. Urinalysis may be helpful and may be indicative of colonization or renal candidiasis. Cultures of nons terile sites, although not useful in establishing a diagnosis, may demonstrate high degrees of candidal colonization. This may be useful in deciding whether to initiate antifungal therapy in patients with fever unresponsive to broadspectrum antimicrobials (khan et al. 1998). Positive blood cultures and cultures from sterile sites, on the other hand, are indicative of definite infection using recently established international definitions of the European Organization for Research and Treatment of Cancer/Mycoses Study Group (EORTC/MSG).

\section{Conclusion}

The epidemiology of candidiasis in Constantine is associated with comorbidity and mortality with patients hospitalized in intensive care units and with high risk factors lesion witch necessity identification and taking in charge rapid Prophylaxis is a general point to prevent an infection.

Notably in candidiasis, it is very effective and based on risk factor identification. However, prophylaxis must be restricted to carefully selected groups of patients, to avoid the emergency of resistant strains and a shift in the distribution of pathogens from albicans to non-albicans strains under the pressure of antifungal agents.

\section{References}

1. Dias MF, Quaresma-Santos MV, Bernardes-Filho F, Amorim AG, Schechtman RC, et al. (2013) Update on therapy for superficial mycoses: review article part I. An Bras Dermatol 88: 764-74.

2. Zai S, Khan SM, Gillani J (1997) Candidiasis in Cancer Patients. J Pak Med Assoc 47: 191-2.

3. Katoh T (2009) Guidelines for diagnosis and treatment of mucocutaneous candidiasis. Nihon Ishinkin Gakkai Zasshi 50: $207-12$.

4. Pappas PG, Rex JH, Sobel JD, Filler SG, Dismukes WE, et al. (2009) Guidelines for Treatment of Candidiasis. Clin Infect Diseas 38: 161-89.

5. Milan EP, Zaror L, Sidrim JJC, Rocha MFG (2004) Leveduras: identificação laboratorial.

6. Sidrim JJC, Rock MFG (2004) Micologia Medica A Luz De Autores Contemporaneos ( $1^{\text {st }}$ Edn) Guanabara Publisher, Italy.

7. Falagas ME, Roussos N, Vardakas KZ (2010) Relative frequency of albicans and the various non-albicans Candida spp among candidemia isolates from inpatients in various parts of the world: a systematic review. Int J Infect Dis 14: e954-66.

8. Anane S, Khalfallah F (2007) Diagnostic biologique des candidoses systémiques : difficultés et perspectives. Pathol Biol 55: 262-72.

9. Matthews RC (1994) Pathogenicity determinants of Candida albicans: potential targets for immunotherapy? Microbiol 140: 1505-11.

10. Lortholary O, Petrikkos G, Akova M, Arendrup MC, Arikan-Akdagli S, et al. (2012) ESCMID* guideline for the diagnosis and management of Candida diseases 2012: patients with HIV infection or AIDS. Clin Microbiol Infect 18: 68-77.

11. Marinho SA, TeixeiraI AB, SantosI OS, CazanovaI RF, FerreiraI CAS, et al. (2010) Identification of Candida spp. by phenotypic tests and PCR. Brazilian J Microbiol dx.doi.org/10.1590/S1517-83822010000200004.

12. Gong YB, Zheng JL, Jin B, Zhuo DX, Huang ZQ, et al. (2012) Particular Candida albicans strains in the digestive tract of dyspeptic patients, identified by multilocus sequence typing. PLoS One 7: doi: 10.1371/journal.pone.0035311. 


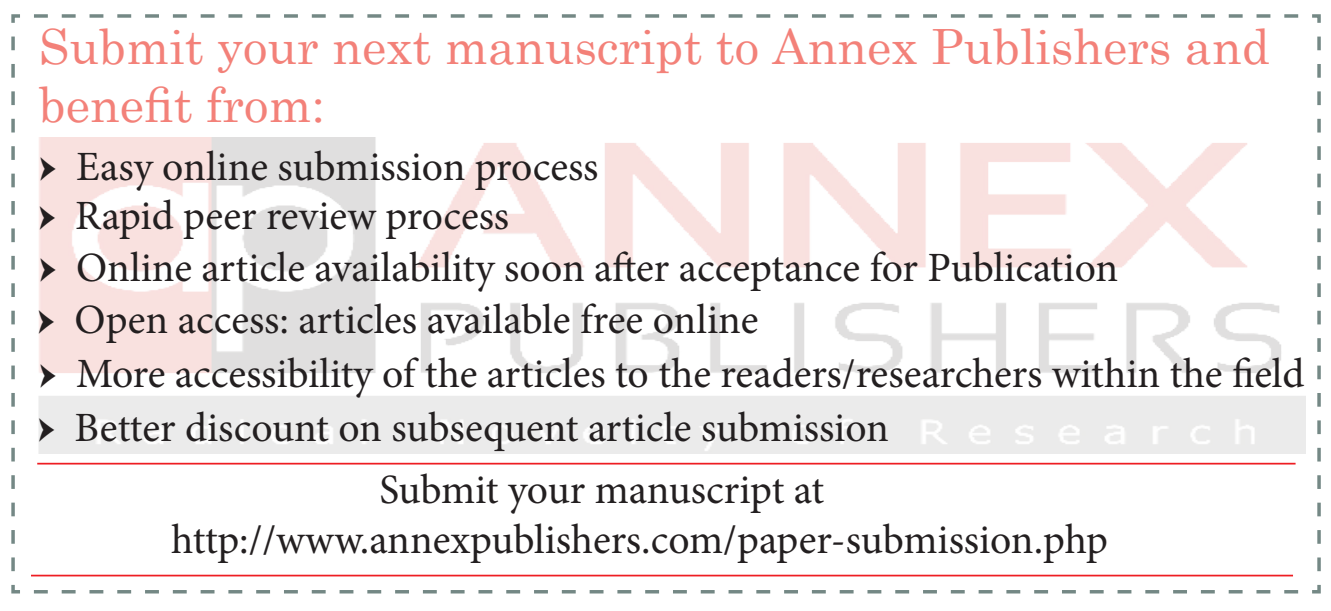

